\title{
Improved Fluid-Model of TCP/AQM Network for Congestion Control
}

\author{
Junsong Wang ${ }^{1 *}$ and Zhiwei Gao ${ }^{2}$ \\ ${ }^{I}$ Center for Intelligent and Networked System, Department of Automation, Tsinghua University, Beijing, 100084, China \\ ${ }^{2}$ School of Electric Engineering and Automation, Tianjin University, China
}

\begin{abstract}
In this paper, recently proposed active queue management (AQM) algorithms for supporting end-to-end transmission control protocol (TCP) congestion control is revisited. We focus recently developed theoretic results on design and analysis for the AQM based TCP congestion control dynamics. In this context, the existing fluid model of the TCP/AQM network is discussed. Moreover, an improved fluid model is addressed, taking time delay in inner feedback loop into account, which is neglected in the modeling process of fluid model. The stabilization of the fluid model is investigated, which has shown that the stabilizing region of PID congestion controller for the conventional fluid model moves $\frac{1}{k}$ and $\frac{T_{1}}{k}$ along the $k_{p}$ and $k_{d}$ axes respectively compared with that for the improved fluid model, ie. the actual stability region. The improved fluid model has a great potential in analyzing and designing various network congestion control algorithms.
\end{abstract}

\section{INTRODUCTION}

State-of-the-art Internet is a decentralized control system employing dynamic Transmission Control Protocols at the sources and Active Queue Management protocols at the routers. The predominant transmission control protocol suites are variants of TCP [1] .TCP flow control is the most important mechanism for congestion control in IP networks. Since Jacobson proposed the end-to-end flow control scheme in 1988 [1], there have been many enhanced and improved versions, such as Tahoe, Reno, New Reno, SACK and Vegas [2-4]. However, these works merely pay attention to the end system. Recent attention has been drawn to explore how to use the intermediate node to avoid congestion because there is a limit to how much control can be accomplished at the end system. AQM [5-7], as one class of packet dropping/marking mechanism in the router queue, has been recently proposed to support end-to-end congestion control in the Internet. It has been a very active research area in the Internet community. The goals of AQM are as follows: (a) to reduce the average length of queues in routers and thereby decrease end-to-end delay experienced by packets; (b) to ensure that network resources are used efficiently by reducing packet loss that occurs when queues overflow. A fluid-flow model of TCP behavior is developed in [8], up to now, which is extensively employed to design various AQM

*Address correspondence to this author at the Center for Intelligent and Networked System, Department of Automation, Tsinghua University, Beijing, 100084, China; Tel: +86-10-62792425; Fax: +86-10-62796115; E-mail; jswang2006@yahoo.com.cn algorithm from the viewpoint of control theory and control engineering $[9,10]$. However, time delay in inner feedback loop is neglected in the modeling process of fluid model, which will result that the stability region derived from the conventional fluid model is not an accurate representation of the actual stability region.

The paper is organized as follows: Sections 2 is devoted to introduce fluid-based network model of TCP/AQM network. An Improved fluid-model of TCP/AQM network is proposed and described in Section 3, including the theoretical analysis and simulation experiments. Finally, Section 4 concludes the paper.

\section{FLUID-BASED NETWORK MODEL OF TCP/AQM NETWORK}

In [8], a dynamic model of TCP behavior was developed using fluid-flow and stochastic differential equation analysis. Simulation results demonstrated that the model accurately captured the dynamics of TCP. In this paper we use a simplified version of that model which ignores the TCP timeout mechanism. This model relates the average value of key network variables and is described by the following coupled, nonlinear differential equations:

$$
\begin{aligned}
& \dot{W}(t)=\frac{1}{R(t)}-\frac{W(t) W(t-R(t))}{2 R(t-R(t))} p(t-R(t)) \\
& \dot{q}(t)=\frac{W(t)}{R(t)} N(t)-C
\end{aligned}
$$


Where $W$ is expected TCP window size (packets), $q$ expected queue length (packets), $C$ link capacity (packets/sec), $R=\frac{q}{C}+T_{p}$ round-trip time (secs), $T_{p}$ propagation delay (secs), $N$ load factor (number of TCP sessions), $p$ probability of packet mark/drop. The first differential equation in (1) describes the TCP window control dynamic. The second equation in (1) models the bottleneck queue length as simply an accumulated difference between packet arrival rate $N W / R$ and link capacity $C$. We illustrate these differential equations in the block diagram of Fig. (1) which highlights TCP window-control and queue dynamics.

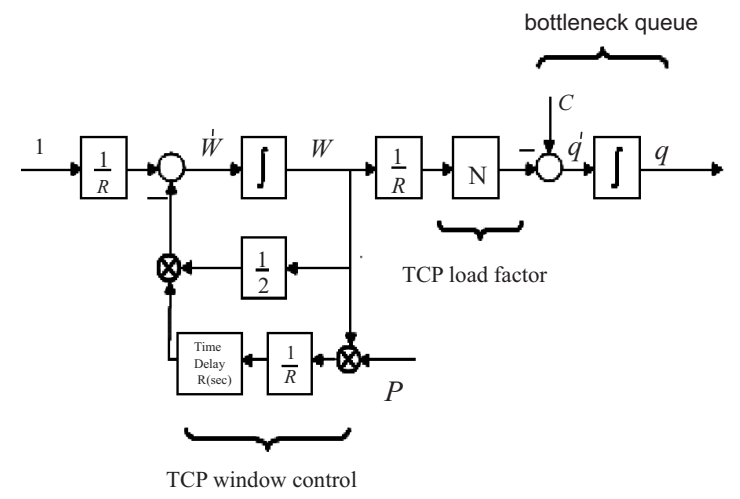

Fig. (1). Block-diagram of TCP's congestion-avoidance flow-control mode.

We now approximate these dynamics by their small-signal linearization about an operating point to gain insight for the purposes of feedback control (AQM). Doing the linearization to (1) about the operating point to give

$\delta \dot{W}(t)=-\frac{2 N}{R_{0}^{2} C} \delta W(t)-\frac{R_{0} C^{2}}{2 N^{2}} \delta p\left(t-R_{0}\right)$

$\delta \dot{q}(t)=\frac{N}{R_{0}} \delta W(t)-\frac{1}{R_{0}} \delta q(t)$

Performing a Laplace transform on the differential equations, the linearized dynamics are illustrated in a block diagram form in Fig. (2).

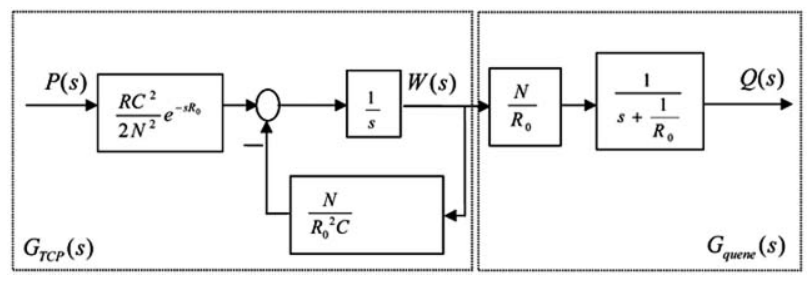

Fg. (2). Block-diagram of the fluid model.
From Fig. (2), one has

$$
G_{T C P}^{\prime}(s)=\frac{W(s)}{P(s)}=\frac{N}{R_{0} s+1},
$$$$
\text { and } G_{\text {queue }}^{\prime}(s)=\frac{Q(s)}{W(s)}=\frac{\frac{\left(R_{0} C\right)^{3}}{4 N^{3}} e^{-R_{0} s}}{\frac{R_{0}^{2} C}{2 N} s+1}
$$

Such that

$$
\begin{aligned}
G_{p}^{\prime}(s) & =\frac{Q(s)}{P(s)}=\frac{W(s)}{P(s)} \cdot \frac{Q(s)}{W(s)} \\
& =G_{T C P}^{\prime}(s) \cdot G_{\text {queue }}^{\prime}(s) \\
& =\frac{k e^{-R_{0} s}}{\left(T_{1} s+1\right)\left(T_{2} s+1\right)}
\end{aligned}
$$

Where $k=\frac{\left(R_{0} C\right)^{3}}{4 N^{2}}, \quad T_{1}=R_{0}$ and $T_{2}=\frac{R_{0}{ }^{2} C}{2 N}$ 。

The fluid model is extensively used in the design of congestion controller for TCP/AQM network [9-13, 14-15].

\section{IMPROVED FLUID-MODEL OF TCP/AQM NET- WORK}

\subsection{Improved Fluid-Model}

It is noticed that time delays in inner feedback loop have been ignored during the linearization process of eqn. 1 about the operating point. Now we can obtain the more accurate linear deferential equations by taking delays into account:

$$
\begin{gathered}
\delta \dot{W}(t)=-\frac{N}{R_{0}^{2} C}\left(\delta W(t)+\delta W\left(t-R_{0}\right)\right) \\
-\frac{R_{0} C^{2}}{2 N^{2}} \delta p\left(t-R_{0}\right) \\
\delta \dot{q}(t)=\frac{N}{R_{0}} \delta W(t)-\frac{1}{R_{0}} \delta q(t)
\end{gathered}
$$

Performing a Laplace transform on eqn.5, the linearized dynamics of the TCP/AQM network are illustrated in a block diagram form in Fig. (3).

From Fig. (3),

$$
\begin{gathered}
G_{T C P}^{\prime}(s)=\frac{W(s)}{P(s)}=\frac{N}{R_{0} s+1}, \\
\text { and } G_{\text {queue }}^{\prime}(s)=\frac{Q(s)}{W(s)}=\frac{\frac{\left(R_{0} C\right)^{3}}{4 N^{3}} e^{-R_{0} s}}{\frac{R_{0}^{2} C}{2 N} s+1+e^{-R_{0} s}}
\end{gathered}
$$

The transfer function of the TCP/AQM network is 


$$
\begin{aligned}
G_{p}(s) & =\frac{Q(s)}{P(s)}=\frac{W(s)}{P(s)} \cdot \frac{Q(s)}{W(s)} \\
& =G_{T C P}(s) G_{\text {quene }}(s) \\
& =\frac{k e^{-R_{0} s}}{\left(T_{1} s+1\right)\left(T_{2} s+1+e^{-R_{0} s}\right)}
\end{aligned}
$$

Where $k=\frac{\left(R_{0} C\right)^{3}}{4 N^{2}}, T_{1}=R_{0}, T_{2}=\frac{R_{0}^{2} C}{2 N}$.

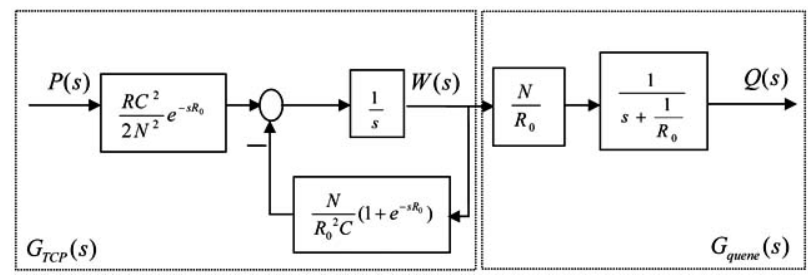

Fig. (3). Block-diagram of the improved fluid model.

\subsection{Stability Analysis of the Improved Fluid-Model}

Lemma 3.1: The plant $G_{p}(s)$ defined in (6) is stable for all positive values of $R_{0} \quad C$ and $N$.

\section{Proof:}

Since

$$
\begin{aligned}
G_{p}(s) & =\frac{k e^{-R_{0} s}}{\left(T_{1} s+1\right)\left(T_{2} s+1+e^{-R_{0} s}\right)} \\
& =\frac{k e^{-R_{0} s}}{\left(T_{1} s+1\right)\left(T_{2} s+1\right)\left(1+\frac{e^{-R_{0} s}}{T_{2} s+1}\right)} \\
& =\frac{k e^{-R_{0} s}}{\left(T_{1} s+1\right)\left(T_{2} s+1\right)} \cdot \frac{1}{1+\frac{e^{-R_{0} s}}{T_{2} s+1}}
\end{aligned}
$$

Apparently, for all positive values of $R_{0}, C$ and $N$, $T_{1}=R_{0}$ and $T_{2}=\frac{R_{0}{ }^{2} C}{2 N}$ are positives, so the zeros of the transfer function $\left(T_{1} s+1\right)$ and $\left(T_{2} s+1\right)$ are in the left-half part of the complex plane for all values of the parameters $R_{0} \quad C$ and $N$.
For $\left(1+\frac{e^{-R_{0} s}}{T_{2} s+1}\right)$, we have

$$
\left|\frac{e^{-R_{0} s}}{T_{2} S+1}\right|_{s=\mathrm{j} \varpi}=\frac{1}{\sqrt{\left(T_{2} \varpi\right)^{2}+1}}<1
$$

According to the Nyquist stability test zero of the transfer function $\left(1+\frac{e^{-R_{0} s}}{T_{2} s+1}\right)$ is also in the left-half part of the complex plane for all values of the parameters $R_{0}, C$ and $N$.

So we can thus conclude that the plant $G_{p}(s)$ defined in (6) is always stable for all positive values of $R_{0}, C$ and $N$.

\subsection{Control Analysis of the Improved Fluid-Model}

The closed-loop Block-diagram of TCP/AQM congestion control system is shown in Fig. (4), then the closed-loop transfer-function of the system becomes

$$
\phi(s)=\frac{C(s) G_{p}(s)}{1+C(s) G_{p}(s)}
$$

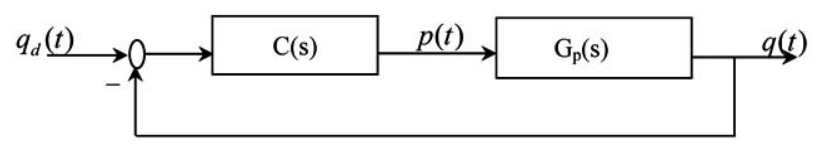

Fig. (4). Block-diagram of the TCP/AQM congestion control system.

Lemma 3.2: For PID controller of TCP/AQM network, the whole size and shape of the stabilizing region of ( $k_{p}, k_{i}, k_{d}$ ) designed based on fluid model is equal to that designed by the improved fluid model, however it moves $\frac{1}{k}$ and $\frac{T_{1}}{k}$ along the $k_{p}$ and $k_{d}$ axes respectively compared with that designed based on the improved fluid model. Since the later more accurately models the dynamics of the TCP/AQM network than the former, which imply that there exists error in the stabilizing region of the PID-type congestion controller designed based on fluid model. 


\section{Proof:}

The transfer function of PID controller is :

$C(s)=k_{p}+\frac{k_{i}}{s}+k_{d} s=\frac{k_{i}+k_{p} s+k_{d} s^{2}}{s}$

where $k_{p}, k_{i}$ and $k_{d}$ are coefficient of proportional, differential and integral respectively.

Upon substitution by (4), the closed-loop characteristic equation of the system shown in Fig. (2) becomes

$\Delta^{\prime}(s)=\left(T_{1} s+1\right)\left(T_{2} s+1\right) s$

$$
+k\left(k_{i}+k_{p} s+k_{d} s^{2}\right) e^{-R_{0} s}
$$

And substitution by (6), the closed-loop characteristic equation of the system shown in Fig. (3) as

$$
\begin{aligned}
\Delta(s)= & \left(T_{1} s+1\right)\left(T_{2} s+1+e^{-R_{0} s}\right) s \\
& +k\left(k_{i}+k_{p} s+k_{d} s^{2}\right) e^{-R_{0} s}
\end{aligned}
$$

Eqn.10 can be rewritten in the form

$$
\begin{aligned}
& \Delta(s)=\left(T_{1} s+1\right)\left(T_{2} s+1\right) s \\
& +k\left[\left(k_{i}+\left(k_{p}+\frac{1}{k}\right) s+\left(k_{d}+\frac{T_{1}}{k}\right) s^{2}\right)\right] e^{-R_{0} s} \\
& =\left(T_{1} s+1\right)\left(T_{2} s+1\right) s+k\left(k_{i}^{\prime}+k_{p}^{\prime} s+k_{d}^{\prime} s^{2}\right) e^{-R_{0} s}
\end{aligned}
$$

Apparently eqn. 11 is the same as eqn.9 in form, where $k_{p}^{\prime}=k_{p}+\frac{1}{k}, k_{i}^{\prime}=k_{i}$ and $k_{d}^{\prime}=k_{d}+\frac{T_{1}}{k}$, is the designed controller's parameter designed based on the fluid model, ie.eqn. 4. $k_{p} 、 k_{i}$ and $k_{d}$ is the designed controller's parameter designed based on the improved fluid model.

For

$k_{p}^{\prime}=k_{p}+\frac{1}{k}, \quad k_{i}^{\prime}=k_{i}, \quad k_{d}^{\prime}=k_{d}+\frac{T_{1}}{k}$

Thus we can conclude that the whole size and shape of the stabilizing region of $\left(k_{p}, k_{i}, k_{d}\right)$ is not changed, but it move $\frac{1}{k}$ and $\frac{T_{1}}{k}$ along the $k_{p}$ and $k_{d}$ axes respectively compared with that designed according to the improved fluid model.
Remark 1 For proportional and proportional-integral congestion controller the whole size and shape of the stabilizing region of $k_{p}$ and $\left(k_{p}, k_{i}\right)$ is not changed, but it move $\frac{1}{k}$ along the $k_{p}$ axes compared with that designed according to the improved fluid model.

Remark 2 since $\frac{1}{k}=\frac{4 N^{2}}{\left(R_{0} C\right)^{3}}$ and $\frac{T_{1}}{k}=\frac{4 N^{2}}{C\left(R_{0} C\right)^{2}}$, so the error of the stabilizing region of congestion controller will become more bigger, with big $N$, and small $R_{0}$ or $C$.

\section{SIMULATION ANALYSIS}

Case 1: The parameter of the TCP/AQM network is equal to that in paper [12] and the expected queue length size is 150 packets so the improved fluid model is

$$
G_{p}(s)=\frac{164.8 e^{-0.0 \nu s}}{(0.05 s+1)\left(0.046875 s+1+e^{-0.05 s}\right)}
$$

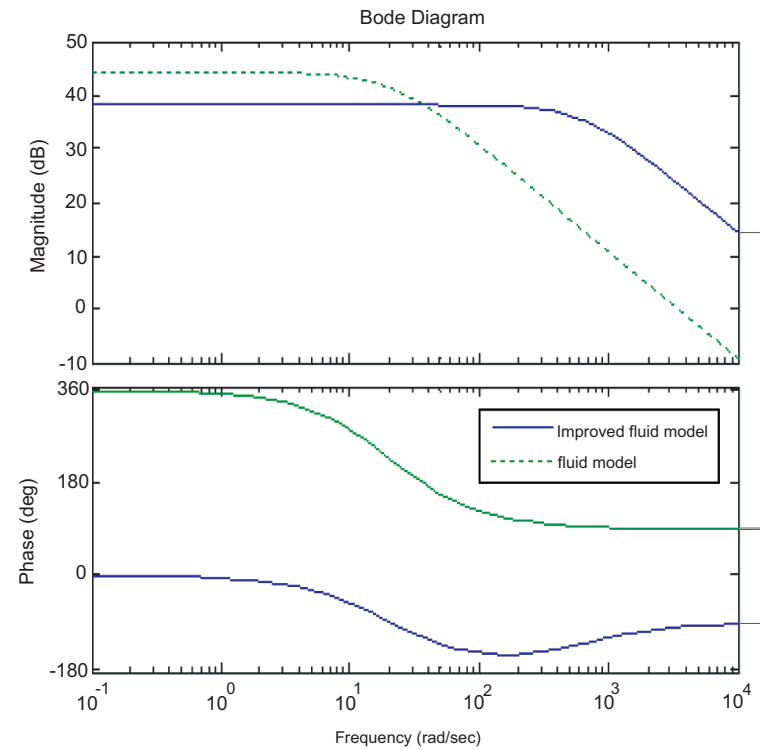

Fig. (5-a). Bode plot of network model in paper [12].

Figs. (5-a) are magnitude plots and phase plots, the solid lines indicate the improved fluid model, while the dash lines show the fluid model. We can see that these two models can not match very well.

The RED congestion controller design in [12] is

$$
C(s)=\frac{5.125 \times 10^{-5}}{0.97745 s+1}
$$

and the simulation result is shown as Fig. (5-b). 


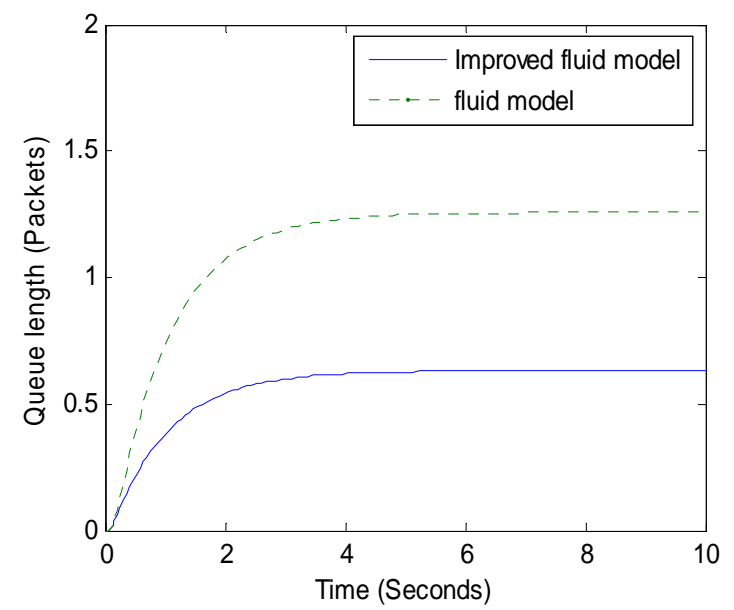

Fig. (5-b). The simulation result of RED congestion controller design in paper [12].

Case 2: the parameter of the TCP/AQM is equal to that in [8], and the expected queue length size 150 is packets, so the improved fluid model is

$$
G_{p}(s)=\frac{54517.6 e^{-0.246 s}}{(0.246 s+1)\left(1.891 s+1+e^{-0.246 s}\right)}
$$
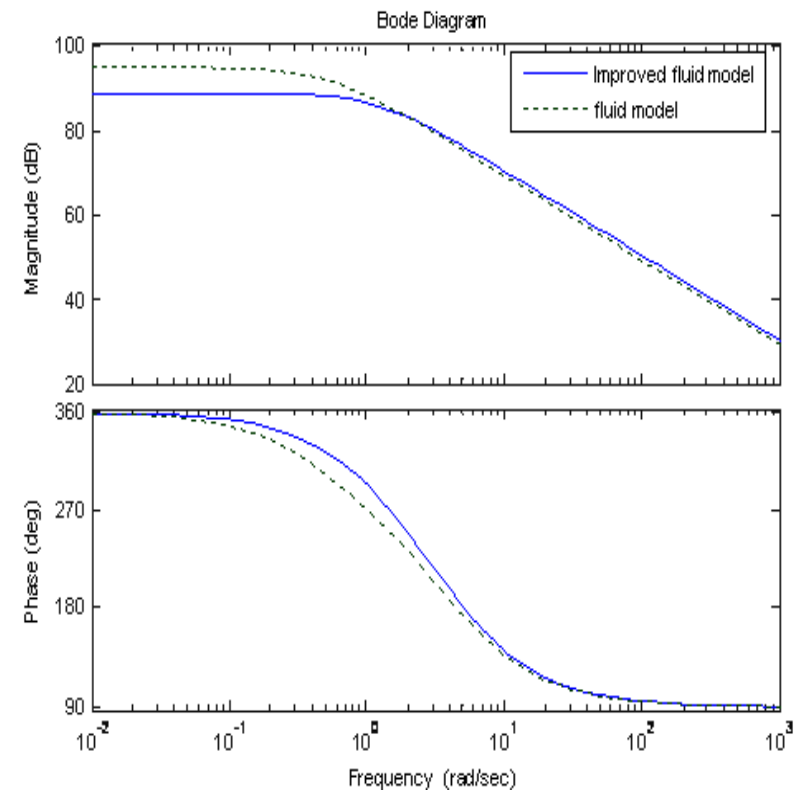

Fig. (6-a). Bode plot of network model in paper [11].

Figs. (6-a) are magnitude plots and phase plots, the solid lines indicate the improved fluid model, while the dash lines show the fluid model. We can see that these two models can wholly match each other.

(1) RED congestion controller:

$$
C(s)=\frac{9.3 \times 10^{-7}}{s+0.005}
$$

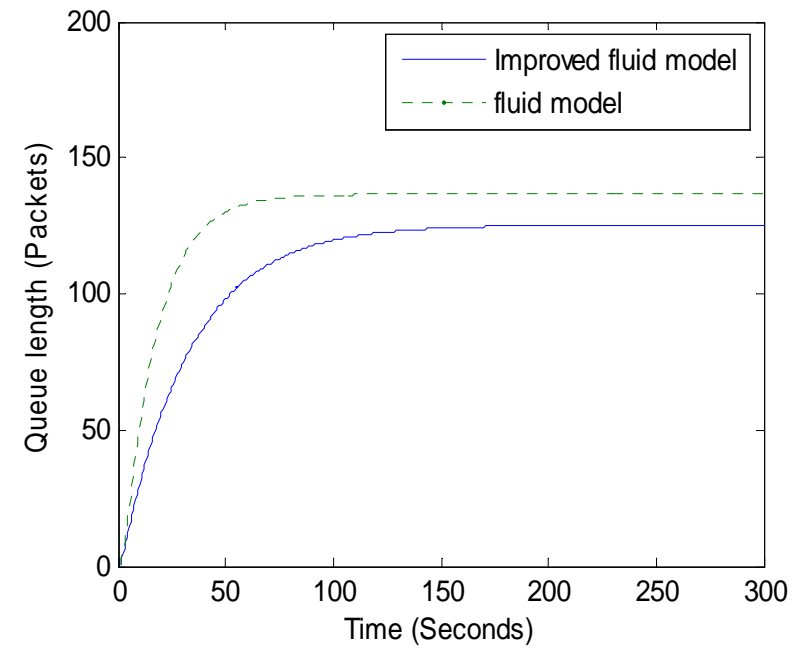

Fig. (6-b). The simulation result of RED congestion controller design in paper [11].

and the simulation result is shown in Fig. (6-b).

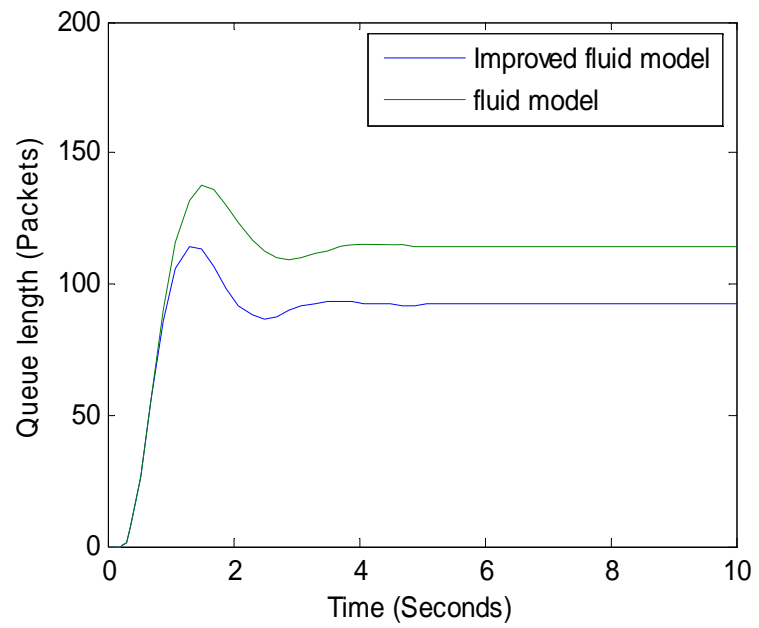

Fig. (6-c). The simulation result of the proportional congestion controller design in paper [11].

(2) Proportional congestion controller:

$$
C(s)=5.8624 \times 10^{-5}
$$

and the simulation result is shown in Fig. (6-c).

(3) Proportional plus integration congestion controller: $C(s)=9.64 \times 10^{-6}\left(1.8868+\frac{1}{s}\right)$, and the simulation result is shown in Fig. (6-d).

Case 3: the parameter of the TCP/AQM is equal to that in [13] and the expected queue length size is 150 packets so the improved fluid model is

$$
G_{p}(s)=\frac{234375 e^{-0.4 s}}{(0.4 s+1)\left(5 s+1+e^{-0.4 s}\right)}
$$




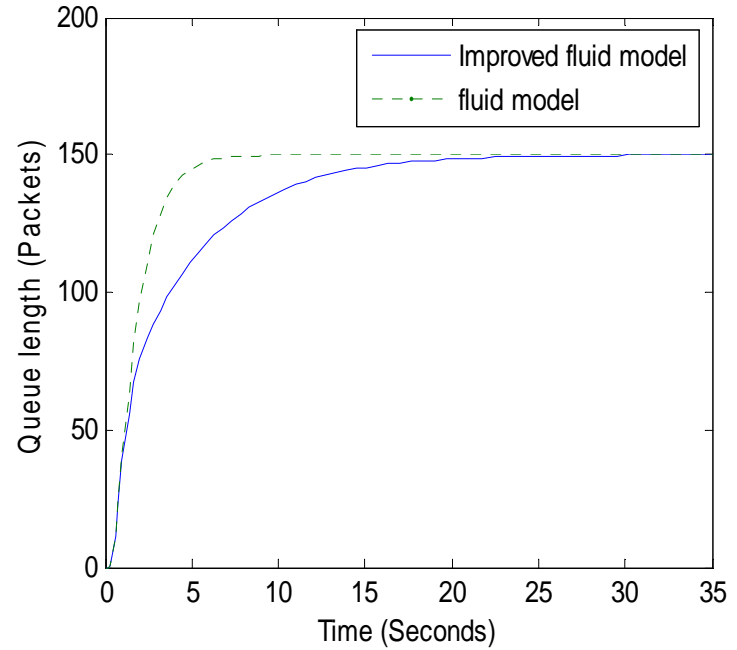

Fig. (6-d). The simulation result of PI controller design in paper [11].

Figs. (7-a) are magnitude plots and phase plots, the solid lines indicate the improved fluid model, while the dash lines show the fluid model. We can see that these two models can wholly match each other.

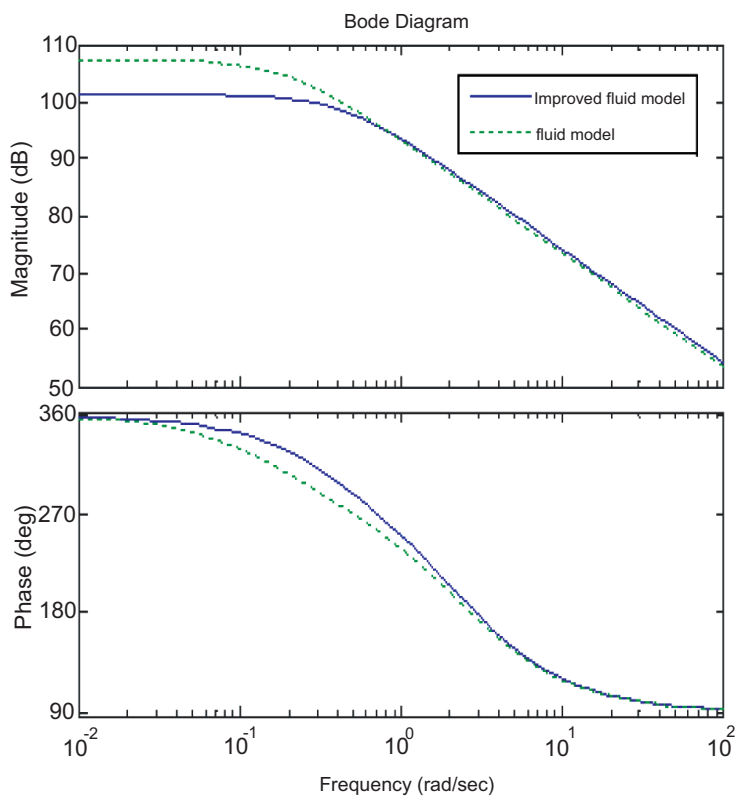

Fig. (7-a). Bode plot of the network model in paper [13].

The controller's parameters is equal to that in paper [13], and the simulation result is shown in Figs. (7-b).

Case 4: The parameter of the TCP/AQM is as $\mathrm{C}=1200$ packets $/ \mathrm{s}, \mathrm{R}=0.01 \mathrm{~s}, \mathrm{~N}=30$, and the expected queue length size is 150 packets so the improved fluid model is

$G_{p}(s)=\frac{0.48 e^{-0.01 s}}{(0.01 s+1)\left(0.002 s+1+e^{-0.01 s}\right)}$

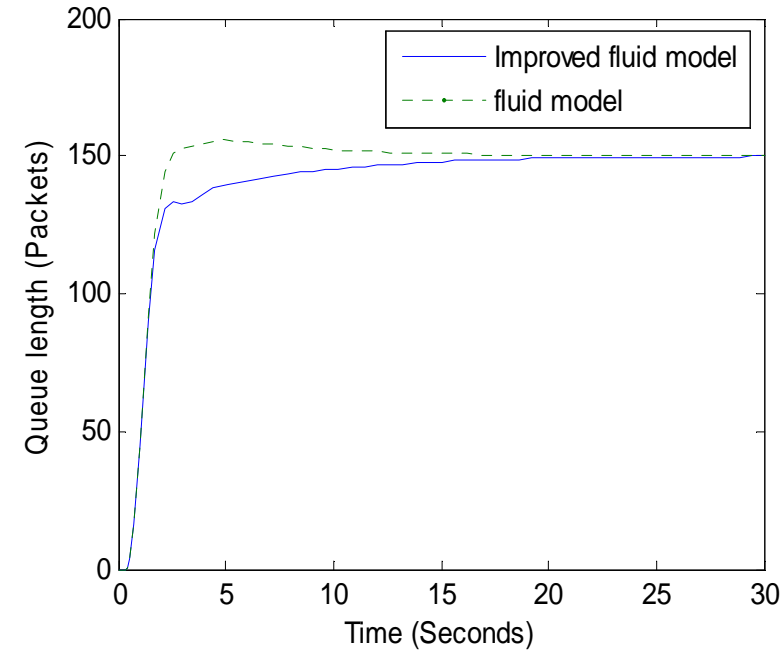

Fig. (7-b). The simulation result of the PID controller design in paper [13].

Figs. (8-a) are magnitude plots and phase plots, the solid lines indicate the improved fluid model, while the dash lines show the fluid model. We can see that these two models can not match each other.

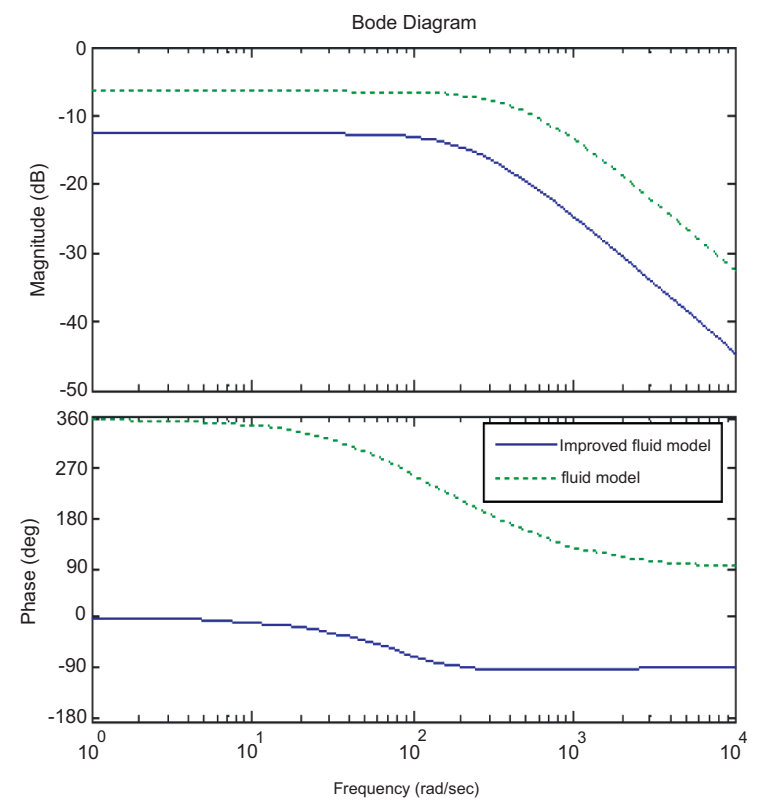

Fig. (8-a). Bode plot of the network model.

The PID controller parameters is $k_{p}=0.5, k_{i}=0.8, k_{d}=0.01$, and the simulation result is shown in Figs. (8-b) and Figs. (8-c).

From the above bode plot based analysis, we can find that the dynamics of the fluid model is different from that of the improved fluid model. Since the later more accurately models the dynamics of the TCP/AQM network than the former, which imply that there exists modeling error in the 


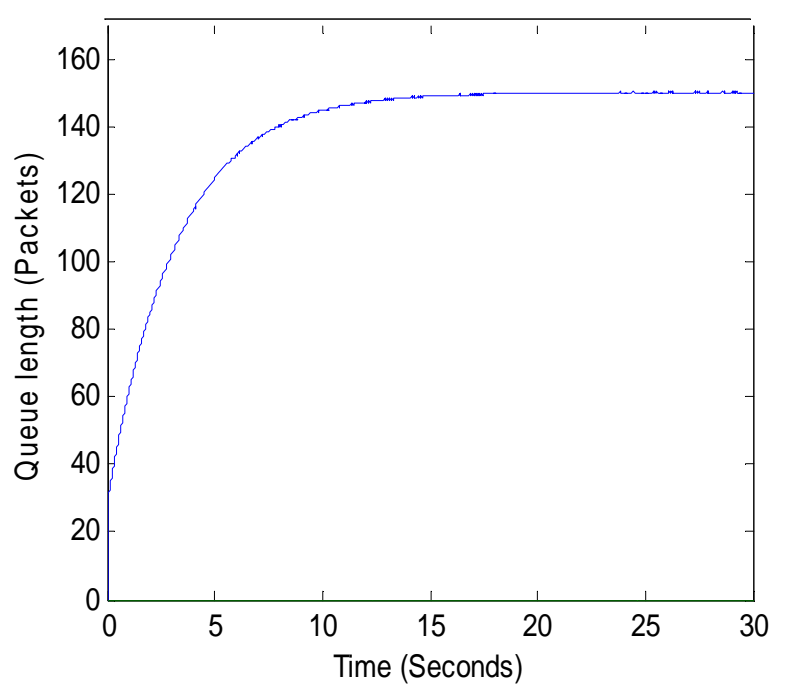

Fig. (8-b). The simulation result of the PID controller by fluid model.

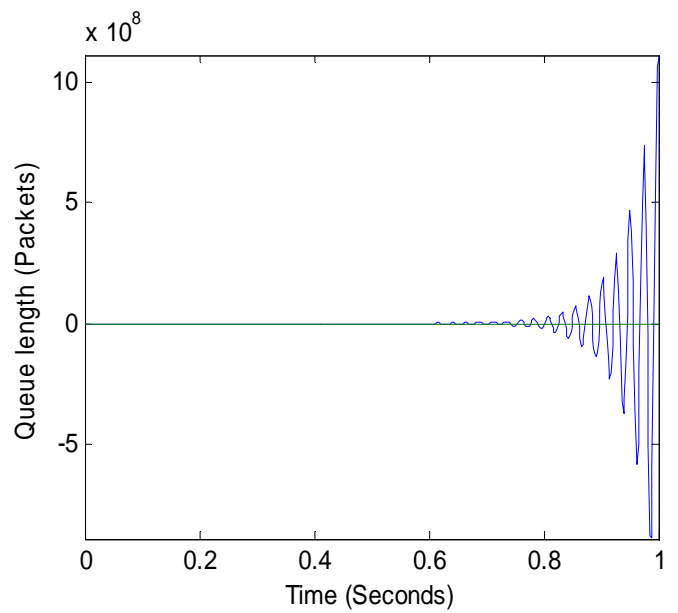

Fig. (8-c). The simulation result of the PID controller by improved fluid model.

former. Furthermore, the simulation results have shown that one congestion controller designed based on fluid model maybe can not obtain satisfactory performance just as Fig. (5-b), Fig. (6-b), Fig. (6-c), Fig. (6-d), Fig. (7-b) and Fig. (8) demonstrated.

\section{CONCLUSION AND DISCUSSION}

In this study, we firstly point out the invalidity of popular fluid model of TCP/AQM network through theoretical analysis and simulation experiments. By considering the time delay in the inner feedback loop, which is neglected in the modeling process of fluid model, we present an improved fluid model. The theoretical analysis has shown that the whole size and shape of the stabilizing region of PID con- gestion controller designed based on fluid model is not changed, but it move $\frac{1}{k}$ and $\frac{T_{1}}{k}$ along $k_{p}$ and $k_{d}$ axes respectively compared with that designed based on the improved fluid model which more accurately represent the dynamic of the TCP/AQM network. This improved fluid model has a great potential in analyzing and designing various network congestion control algorithms.

\section{NOMENCLATURE}

$\begin{array}{lll}\mathrm{TCP} & = & \text { Transmission Control Protocols } \\ \mathrm{AQM} & = & \text { Active Queue Management }\end{array}$

\section{REFERENCES}

[1] V. Jacobson, "Congestion avoidance and control," in Proc. ACM SIGCOMM, 1988, pp. 314-329.

[2] W.R. Stevens, "TCP Slow Start, Congestion Avoidance, Fast Retransmit, and Fast Recovery Algorithms', Internet RFC 2001, January 1997.

[3] K. Fall, and S. Floyd, "Simulation-based comparisons of Tahoe, Reno, and SACK TCP', Computer Communication Review, vol. 26, no. 3, pp. 5-21, 1996.

[4] L. Brakmo, and L. Peterson, "TCP Vegas: end-to-end congestion avoidance on a global Internet', IEEE Journal on Selected Areas in Communication, vol. 13, no. 8, pp. 1465-1480, 1995.

[5] S. Floyd and V. Jacobson, "Random early detection gateways for congestion avoidance," IEEE/ACM Transaction Networking, vol. 1, pp. 397-413, Aug. 1993.

[6] T. Bonald, M. May, and J. C. Bolot, "Analytic evaluation of RED performance," in Proceedings IEEE INFOCOM, 2000, pp. 1415-1424.

[7] M. Christiansen, K. Jeffay, D. Ott, and F. D. Smith, "Tuning RED for web traffic," in Proc. ACM SIGCOMM, 2000, pp. 139-150.

[8] V. Misra, W.-B. Gong, and D. Towsley, "Fluid-based analysis of a network of AQM routers supporting TCP flows with an application to RED," in Proc. ACM SIGCOMM, 2000, pp. 151-160.

[9] C. V. Hollot, V. Misra, D. Towsley, and W.-B. Gong, "On designing improved controllers for AQM routers supporting TCP flows," in Proc. IEEE/INFOCOM, 2001, pp. 1726-1734.

[10] C. V. Hollot, V. Misra, D. Towsley, andW.-B. Gong, "A control theoretic analysis of RED," in Proc. IEEE/INFOCOM, 2001, pp. 1510-1519

[11] C. V. Hollot, V. Misra, D. Towsley, and W. B. Gong, "Analysis and design of controllers for AQM routers supporting TCP flows", IEEE Transactions on Automatic Control, vol. 47, no. 3, pp. 945-959, 2002.

[12] L. Tan, G. Peng, and G. Chen, "Stability of TCP/RED systems in AQM routers", IEEE Transactions on Automatic Control, vol. 51, no. 8, pp. 1393-1398, 2006.

[13] F. Ren, C Lin, B. Wei, "A robust active queue management algorithm in large delay networks", Computer Communications, vol. 28, pp. 485-493, 2005

[14] J. Wang, Z. Gao and N. Xu, "A novel associative memory system based modeling and prediction of TCP network traffic", Lecture Notes on Computer Science, vol. 4491, 519-527, 2007.

[15] J. Wang, Z. Gao, and Y. Shu, "RBF-PID based adaptive active queue management algorithm for TCP network', 2007 IEEE international conference on control and automation, May, 2007.

(C) Wang and Gao; Licensee Bentham Open.

This is an open access article licensed under the terms of the Creative Commons Attribution Non-Commercial License (http://creativecommons.org/licenses/by-nc/3.0/) which permits unrestricted, non-commercial use, distribution and reproduction in any medium, provided the work is properly cited. 